\title{
On the relation between RG and ERG
}

\author{
H Sonoda \\ Physics Department, Kobe University, Kobe 657-8501, Japan \\ E-mail: hsonoda@kobe-u.ac.jp
}

\begin{abstract}
We discuss how the ordinary renormalization group (RG) equations arise in the context of Wilson's exact renormalization group (ERG) as formulated by Polchinski. We consider the $\phi^{4}$ theory in four dimensional euclidean space as an example, and introduce a particular scheme of parameterizing the solutions of the ERG equations. By analyzing the scalar composite operators of dimension two and four, we show that the parameters obey mass independent RG equations. We conjecture the equivalence of our parameterization scheme with the MS scheme for dimensional regularization.
\end{abstract}

PACS numbers: 11.10.Gh, 11.10.Hi

Submitted to: J. Phys. A: Math. Gen. 


\section{Introduction}

The exact renormalization group (ERG) was introduced by K. G. Wilson as a proper language to define continuum limits in quantum field theory. [1] A key ingredient is the theory space $\mathcal{S}$. Given a set of fields, it consists of all possible theories (i.e., lagrangians or actions) with the same cutoff (or defined on the same lattice). A renormalization group $(\mathrm{RG})$ transformation acts on $\mathcal{S}$, and it consists of two steps:

(i) integrating out high momentum modes

(ii) rescaling space to restore the same cutoff

We incorporate short-distance physics into the action, leaving long-distance physics for further integration of field variables. Starting from a theory, RG transformations generate a flow of theories along which the same physics is kept. Only the physical momentum scale of the cutoff becomes smaller along the flow. In this setup, the continuum limits form a finite dimensional subspace $\mathcal{S}(\infty)$ of $\mathcal{S}$. It is centered around a fixed point, and its dimension is given by the number of relevant (or renormalized) parameters. The continuum limits can be obtained as the long distance limit of theories finely tuned to criticality. Using physical units, this prescription defines the limit of the infinite UV cutoff. RG transformations of the renormalized parameters are obtained simply by restricting ERG on $\mathcal{S}(\infty)$.

It was Polchinski who first introduced ERG into perturbative field theory. 2] Polchinski's ERG gives a concrete realization of Wilson's ERG, but it differs from Wilson's in two aspects:

(i) no rescaling of space

(ii) artificial splitting of the action into the free and interacting parts

The first point is not essential since we can easily modify Polchinski's ERG to incorporate rescaling. The second point is an unavoidable nature of perturbation theory.

In fact a more serious difference lies elsewhere. To be concrete, let us consider $\phi^{4}$ theory in four dimensional euclidean space. The (perturbatively) renormalized theory has two parameters, which we can take as a squared mass $m^{2}$ and a coupling $\lambda$. Hence, $\mathcal{S}(\infty)$ is two dimensional, and therefore we need only one parameter to distinguish various ERG flows. But this is not the case in Polchinski's ERG. In sect. 4 we will

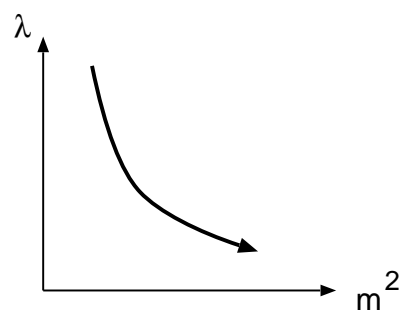

Figure 1. Wilson's ERG: only one parameter to specify an ERG flow 
show that the action satisfying Polchinski's ERG differential equation depends on three parameters $\left(m^{2}, \lambda ; \mu\right)$ :

$$
S\left(\Lambda ; m^{2}, \lambda ; \mu\right)
$$

where $\Lambda$ is the cutoff, decreasing along each ERG flow. The momentum scale $\mu$ is introduced as the scale where $m^{2}, \lambda$ are defined. The two flows

$$
\left(m^{2}, \lambda ; \mu\right) \quad \& \quad\left(m^{2} \mathrm{e}^{2 t}, \lambda ; \mu \mathrm{e}^{t}\right)
$$

correspond to different choices of mass units, and they are trivially equivalent. Let us denote the beta function of $\lambda$ as $\beta(\lambda)$, and the anomalous dimension of $m^{2}$ as $\beta_{m}(\lambda)$. Then, for an infinitesimal $\Delta t$, the flow

$$
\left(m^{2}\left(1+\Delta t \beta_{m}(\lambda)\right), \lambda(1+\Delta t \beta(\lambda)) ; \mu \mathrm{e}^{-\Delta t}\right)
$$

is physically equivalent to the flow $\left(m^{2}, \lambda ; \mu\right)$, but the problem is that the two solutions

$$
S\left(\Lambda ; m^{2}, \lambda ; \mu\right) \quad \& \quad S\left(\Lambda ; m^{2}\left(1+\Delta t \beta_{m}\right), \lambda+\Delta t \beta ; \mu(1-\Delta t)\right)
$$

do not overlap. It is easy to see why. The ERG differential equation depends on $m^{2}$ explicitly, and the two solutions solve two different ERG differential equations. It is

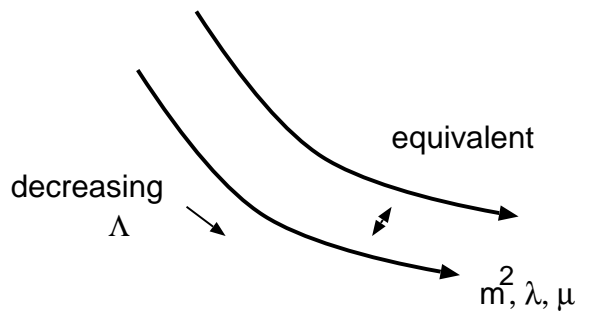

Figure 2. Polchinski's ERG: three parameters to specify an ERG flow

this redundancy of parameters which characterizes Polchinski's ERG. This makes the derivation of $\beta, \beta_{m}$ not straightforward for Polchinski's ERG.

The purpose of this paper is twofold. First we show how a momentum scale $\mu$ enters the solution of Polchinski's ERG equations. This is done in sects. 2, 3, 4. Second we derive $\beta, \beta_{m}$, and the anomalous dimension $\gamma$ of the scalar field in the framework of Polchinski's ERG. Our derivation relies on the technique of composite operators. By studying the $\mu$ dependence of the action, we will show how $\beta, \beta_{m}, \gamma$ arise naturally from ERG. All this is done in sects. 5, 6, 7.

Not long after Polchinski's work, Hughes and Liu have looked at the relation between ERG and RG. [3] Besides some technicalities, the main difference from the present work is that they have overlooked the difference between Wilson's and Polchinski's ERG. This neglect makes their results valid only at the lowest non-trivial orders in perturbation theory.

In an unpublished work [4] we have obtained the same results for $\beta, \beta_{m}, \gamma$ using Polchinski's ERG. The present work is based upon an entirely different technique of composite operators which, the author hopes, makes the paper easier to follow. 


\section{Polchinski's formulation of Wilson's exact renormalization group}

We consider a real scalar field theory in four dimensional euclidean space. Let $S$ be the action so that the correlation functions are given in the momentum space as

$$
\begin{gathered}
\left\langle\phi\left(p_{1}\right) \cdots \phi\left(p_{2 n}\right)\right\rangle_{S} \cdot(2 \pi)^{4} \delta^{(4)}\left(p_{1}+\cdots+p_{2 n}\right) \\
\equiv\left(\int[d \phi] \mathrm{e}^{S}\right)^{-1} \int[d \phi] \phi\left(p_{1}\right) \cdots \phi\left(p_{2 n}\right) \mathrm{e}^{S}
\end{gathered}
$$

$S$ consists of a free part $S_{\text {free }}$ and an interaction part $S_{\text {int }}$ :

$$
S=S_{\text {free }}+S_{\text {int }}
$$

where

$$
\begin{aligned}
& S_{\text {free }} \equiv-\frac{1}{2} \int_{p} \phi(p) \phi(-p) \frac{p^{2}+m^{2}}{K(p / \Lambda)} \quad\left(\int_{p} \equiv \int \frac{d^{4} p}{(2 \pi)^{4}}\right) \\
& S_{\text {int }} \equiv \sum_{n=1}^{\infty} \frac{1}{(2 n) !} \int_{p_{1}, \cdots, p_{2 n}} \phi\left(p_{1}\right) \cdots \phi\left(p_{2 n}\right) \\
& \quad \times(2 \pi)^{4} \delta^{(4)}\left(p_{1}+\cdots+p_{2 n}\right) \cdot \mathcal{V}_{2 n}\left(\Lambda ; p_{1}, \cdots, p_{2 n}\right)
\end{aligned}
$$

We take the cutoff function $K(x)$ as a decreasing positive function of $x^{2}$ with the properties that $K(x)=1$ for $0 \leq x^{2} \leq 1$, and that it decreases sufficiently fast for large $x^{2} \gg 1$. (See the left in Fig. 3.) $\Lambda$ is the momentum cutoff, suppressing the field
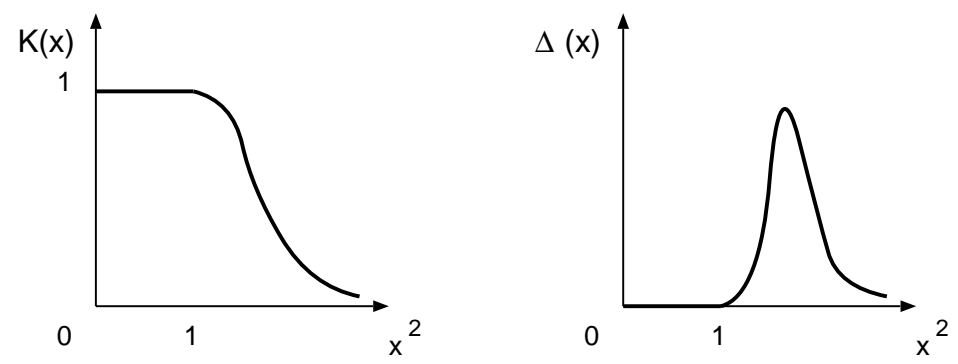

Figure 3. The behavior of the cutoff function $K$ and its derivative $\Delta$

fluctuations of momenta larger than $\Lambda$. For convenience, we introduce

$$
\Delta(x) \equiv-2 x^{2} \frac{d K(x)}{d x^{2}}
$$

This vanishes for $0 \leq x^{2}<1$, and is positive for $x^{2}>1$. (See the right in Fig. 3.)

Given an action $S$, the correlation functions can be computed perturbatively using

$$
\frac{K(p / \Lambda)}{p^{2}+m^{2}}
$$

as the propagator, and $\mathcal{V}_{2 n}$ as the interaction vertices. Thanks to the rapid decrease of $K(p / \Lambda)$ for $p^{2} \gg \Lambda^{2}$, the Feynman integrals are free from ultraviolet divergences. The use of a finite ultraviolet cutoff $\Lambda$ is compensated, however, by the presence of an infinite number of interaction terms. Each interaction vertex $\mathcal{V}_{2 n}$ results from the integration over field momenta larger than $\Lambda$, and it is local in the sense that it can be expanded in 
powers of $m^{2}$ and external momenta if they are small compared to appropriate powers of $\Lambda$. In the extreme limit $\Lambda \rightarrow 0, \mathcal{V}_{2 n}$ reduces to the connected $2 n$-point correlation function.

As we change $\Lambda$, we wish to change $S(\Lambda)$ so that physics is preserved. This requirement translates into Polchinski's differential equation [2]

$$
\begin{aligned}
-\Lambda \frac{\partial}{\partial \Lambda} S(\Lambda)=\int_{p} \frac{\Delta(p / \Lambda)}{p^{2}+m^{2}} & {\left[\frac{p^{2}+m^{2}}{K(p / \Lambda)} \phi(p) \frac{\delta S(\Lambda)}{\delta \phi(p)}\right.} \\
+ & \left.\frac{1}{2}\left(\frac{\delta S(\Lambda)}{\delta \phi(p)} \frac{\delta S(\Lambda)}{\delta \phi(-p)}+\frac{\delta^{2} S(\Lambda)}{\delta \phi(p) \delta \phi(-p)}\right)\right]
\end{aligned}
$$

This equation assures that the correlation functions, calculated with $S(\Lambda)$, are independent of $\Lambda$. To be more precise, the following combinations do not depend on $\Lambda$ :

$$
\frac{1-(K(p / \Lambda))^{-1}}{p^{2}+m^{2}}+\langle\phi(p) \phi(-p)\rangle_{S(\Lambda)} \frac{1}{K(p / \Lambda)^{2}}
$$

for the two-point, and

$$
\left\langle\phi\left(p_{1}\right) \cdots \phi\left(p_{2 n}\right)\right\rangle_{S(\Lambda)} \prod_{i=1}^{2 n} \frac{1}{K\left(p_{i} / \Lambda\right)}
$$

for the higher-point functions $n>1$. Despite the use of a finite ultraviolet cutoff, the action can contain physics of momentum scales up to the highest $\Lambda$ for which $S$ is well defined.

\section{Solution by initial conditions}

A standard way of solving (11) is by imposing a set of initial conditions at a large cutoff $\Lambda_{0}$. In 2] Polchinski showed the possibility of choosing initial conditions such that $S(\Lambda)$, for any finite $\Lambda$, has a limit as $\Lambda_{0} \rightarrow \infty$. (This was shown, of course, within perturbation theory.) For example, we can adopt the following mass independent form:

$$
\begin{aligned}
& \mathcal{V}_{2}\left(\Lambda_{0} ; p,-p\right) \\
& \quad=\Lambda_{0}^{2} g\left(\lambda, \ln \left(\Lambda_{0} / \mu\right)\right)+m^{2} z_{m}\left(\lambda, \ln \left(\Lambda_{0} / \mu\right)\right)+p^{2} z\left(\lambda, \ln \left(\Lambda_{0} / \mu\right)\right) \\
& \mathcal{V}_{4}\left(\Lambda_{0} ; p_{1}, \cdots, p_{4}\right)=-\lambda\left\{1+z_{\lambda}\left(\lambda, \ln \left(\Lambda_{0} / \mu\right)\right)\right\} \\
& \mathcal{V}_{2 n \geq 6}\left(\Lambda_{0} ; p_{1}, \cdots, p_{2 n}\right)=0
\end{aligned}
$$

$g, z_{m}, z$, and $z_{\lambda}$ are all given as power series in $\lambda$ whose coefficients depend logarithmically on $\Lambda_{0}$. Here $\mu$ is an arbitrary finite momentum scale. It is not only necessary to render the argument of the logarithm dimensionless, but it also acquires an important physical interpretation as the scale where the renormalized parameters $\lambda, m^{2}$ are defined.

To understand better how $\mu$ enters the continuum limit, let us look at the one-loop two-point vertex. Solving (11) we obtain

$$
\begin{aligned}
& \mathcal{V}_{2}(\Lambda ; p,-p)-\mathcal{V}_{2}\left(\Lambda_{0} ; p,-p\right) \\
& =\frac{-\lambda}{4}\left(\Lambda_{0}^{2}-\Lambda^{2}\right) \int_{q} \frac{\Delta(q)}{q^{2}}+\frac{\lambda}{(4 \pi)^{2}} m^{2} \ln \left(\Lambda_{0} / \Lambda\right)
\end{aligned}
$$




$$
-\frac{\lambda}{2} \int_{\Lambda}^{\Lambda_{0}} \frac{d \Lambda^{\prime}}{\Lambda^{\prime}} \int_{q} \Delta\left(q / \Lambda^{\prime}\right) \frac{m^{4}}{q^{4}\left(q^{2}+m^{2}\right)}
$$

where the last double integral on the right-hand side is finite as $\Lambda_{0} \rightarrow \infty$. Therefore, for $\mathcal{V}_{2}(\Lambda ; p,-p)$ to have a finite limit as $\Lambda_{0} \rightarrow \infty$, we can take

$$
\begin{aligned}
& g\left(\lambda, \ln \left(\Lambda_{0} / \mu\right)\right)=\frac{\lambda}{4} \int_{q} \frac{\Delta(q)}{q^{2}} \\
& z_{m}\left(\lambda, \ln \left(\Lambda_{0} / \mu\right)\right)=-\frac{\lambda}{(4 \pi)^{2}} \ln \left(\Lambda_{0} / \mu\right) \\
& z\left(\lambda, \ln \left(\Lambda_{0} / \mu\right)\right)=0
\end{aligned}
$$

so that

$$
\begin{aligned}
\mathcal{V}_{2}(\Lambda ; p,-p)= & \frac{\lambda}{4} \Lambda^{2} \int_{q} \frac{\Delta(q)}{q^{2}}-\frac{\lambda}{(4 \pi)^{2}} m^{2} \ln (\Lambda / \mu) \\
& -\frac{\lambda}{2} \int_{\Lambda}^{\infty} \frac{d \Lambda^{\prime}}{\Lambda^{\prime}} \int_{q} \Delta\left(q / \Lambda^{\prime}\right) \frac{m^{4}}{q^{4}\left(q^{2}+m^{2}\right)}
\end{aligned}
$$

in the continuum limit. Note that $\mathcal{V}_{2}\left(\Lambda_{0} ; p,-p\right)$ cannot depend on $\Lambda$ since it gives an initial condition to the differential equation (11) which determines the $\Lambda$ dependence of $\mathcal{V}_{2}(\Lambda ; p,-p)$. We are thus obliged to introduce $\mu$ to make sense of the logarithm of $\Lambda_{0}$ for $z_{m}$. Similarly, the choice

$$
z_{\lambda}\left(\lambda, \ln \left(\Lambda_{0} / \mu\right)\right)=\frac{3 \lambda}{(4 \pi)^{2}} \ln \left(\Lambda_{0} / \mu\right)
$$

makes the continuum limit of $\mathcal{V}_{4}(\Lambda)$ finite. Arbitrary finite constants can be added to $z_{m}, z_{\lambda}, z$, amounting to further finite renormalization.

The dependence of the theory on an arbitrary momentum scale $\mu$ is familiar from the standard renormalization theory. In the above we have adopted a minimal scheme in which the expansions of $z_{m}, z_{\lambda}, z$ have at least one power of $\ln \left(\Lambda_{0} / \mu\right)$, with no part independent of $\ln \left(\Lambda_{0} / \mu\right)$. Giving a different value to $\mu$ amounts to finite renormalization. This permits us to interpret $\mu$ as the scale where the renormalized parameters $m^{2}, \lambda$ are defined. Unless we choose to parameterize $S(\Lambda)$ in terms of physical parameters such as a physical squared mass and a physical coupling, the introduction of $\mu$ is inevitable.

\section{Solution by asymptotic conditions}

There is another way of solving (11), which is more convenient for relating ERG to the $\mathrm{RG}$ of renormalized parameters.

We first note that perturbative renormalizability amounts to

$$
\mathcal{V}_{2 n}\left(\Lambda ; p_{1}, \cdots, p_{2 n}\right) \stackrel{\Lambda \rightarrow \infty}{\longrightarrow} 0
$$

for $2 n \geq 6$. Besides the squared mass $m^{2}$ that appears in the ERG equation (111) itself, the solutions depend on a coupling parameter $\lambda$. Hence, we can write the vertices in the form

$$
\begin{aligned}
& \mathcal{V}_{2 n}\left(\Lambda ; p_{1}, \cdots, p_{2 n}\right) \\
& \quad=\Lambda^{y_{2 n}} v_{2 n}\left(\ln (\Lambda / \mu) ; p_{1} / \Lambda, \cdots, p_{2 n} / \Lambda ; m^{2} / \Lambda^{2}, \lambda\right)
\end{aligned}
$$


where

$$
y_{2 n} \equiv 4-2 n
$$

and $\mu$ is a momentum scale introduced to characterize the asymptotic behavior of the vertices. Expanding in powers of $m^{2} / \Lambda^{2}$ and $p^{2} / \Lambda^{2}$ we obtain

$$
\begin{aligned}
& \mathcal{V}_{2}(\Lambda ; p,-p) \\
& \quad=\Lambda^{2} a_{2}(\ln (\Lambda / \mu) ; \lambda)+m^{2} b_{2}(\ln (\Lambda / \mu) ; \lambda)+p^{2} c_{2}(\ln (\Lambda / \mu) ; \lambda)+\cdots
\end{aligned}
$$

Similarly, we obtain

$$
\mathcal{V}_{4}\left(\Lambda ; p_{1}, \cdots, p_{4}\right)=a_{4}(\ln (\Lambda / \mu) ; \lambda)+\cdots
$$

The parts represented by dots are proportional to inverse powers of $\Lambda$, and vanish in the limit $\Lambda \rightarrow \infty$.

At each order of $\lambda$, the asymptotic parts of the vertices are given as finite degree polynomials of $\ln (\Lambda / \mu)$. The coefficients of a polynomial, say $P(\ln (\Lambda / \mu))$, depend on the choice of $\mu$. For example, the coefficient of the constant term, $P(0)$, can be made to vanish by choosing a particular value for $\mu$.

The ERG differential equation specifies only the $\Lambda$ dependence of the vertices. Hence, the $\Lambda$ independent parts of $b_{2}, c_{2}, a_{4}$ do not get fixed uniquely. One way of removing the ambiguity is to adopt the following conditions [5]:

$$
\begin{aligned}
& b_{2}(0 ; \lambda)=c_{2}(0 ; \lambda)=0 \\
& a_{4}(0 ; \lambda)=-\lambda
\end{aligned}
$$

We call this MS (minimal subtraction), since it resembles the MS scheme for dimensional regularization. The $1 / \epsilon$ in dimensional regularization corresponds to $\ln (\Lambda / \mu)$. (28, 29) are the obvious analogues of the absence of finite parts in $\epsilon$ in the three renormalization constants.

Adopting MS, straightforward one-loop calculations give the following results:

$$
\begin{aligned}
\mathcal{V}_{2}(\Lambda ; p,-p)= & \frac{\lambda}{4} \Lambda^{2} \int \frac{\Delta(q)}{q^{2}}-\frac{\lambda}{(4 \pi)^{2}} m^{2} \ln (\Lambda / \mu) \\
& -\frac{\lambda}{2}\left(m^{2}\right)^{2} \int_{\Lambda}^{\infty} \frac{d \Lambda^{\prime}}{\Lambda^{\prime 3}} \int_{q} \frac{\Delta(q)}{q^{4}\left(q^{2}+m^{2} / \Lambda^{\prime 2}\right)}
\end{aligned}
$$

for the two-point vertex (this is the same as (21) ), corresponding to

$$
b_{2}(\ln (\Lambda / \mu) ; \lambda)=-\frac{\lambda}{(4 \pi)^{2}} \ln (\Lambda / \mu), \quad c_{2}=0
$$

and

$$
\begin{aligned}
& \mathcal{V}_{4}\left(\Lambda ; p_{1}, \cdots, p_{4}\right)=-\lambda-\lambda \sum_{i=1}^{4} \frac{1-K\left(p_{i} / \Lambda\right)}{p_{i}^{2}+m^{2}} \mathcal{V}_{2}\left(\Lambda ; p_{i},-p_{i}\right) \\
& +\frac{\lambda^{2}}{2} \int_{q}\left[\frac{1-K(q / \Lambda)}{q^{2}+m^{2}} \frac{1-K\left(\left(q+p_{1}+p_{2}\right) / \Lambda\right)}{\left(q+p_{1}+p_{2}\right)^{2}+m^{2}}-\frac{(1-K(q / \Lambda))^{2}}{q^{4}}\right] \\
& +(\mathrm{t}, \text { u-channels })-3 \frac{\lambda^{2}}{(4 \pi)^{2}} \ln (\Lambda / \mu)
\end{aligned}
$$


for the four-point vertex, corresponding to

$$
a_{4}(\ln (\Lambda / \mu) ; \lambda)=-\lambda\left(1+\frac{3 \lambda}{(4 \pi)^{2}} \ln (\Lambda / \mu)\right)
$$

In our MS scheme the action depends on the four parameters $m^{2}, \lambda, \mu, \Lambda$, and we may denote the action as

$$
S\left(\Lambda ; m^{2}, \lambda ; \mu\right)
$$

Using the cutoff function, we can define the renormalized correlation functions

$$
\begin{aligned}
& \langle\phi(p) \phi(-p)\rangle_{m^{2}, \lambda ; \mu} \equiv \frac{1-(K(p / \Lambda))^{-1}}{p^{2}+m^{2}}+\langle\phi(p) \phi(-p)\rangle_{S(\Lambda)} \frac{1}{K(p / \Lambda)^{2}} \\
& \left\langle\phi\left(p_{1}\right) \cdots \phi\left(p_{2 n}\right)\right\rangle_{m^{2}, \lambda ; \mu} \equiv\left\langle\phi\left(p_{1}\right) \cdots \phi\left(p_{2 n}\right)\right\rangle_{S(\Lambda)} \prod_{i=1}^{2 n} \frac{1}{K\left(p_{i} / \Lambda\right)}
\end{aligned}
$$

which are independent of $\Lambda$. Let us recall that in the MS scheme for dimensional regularization, the $\mu$ dependence of the the correlation functions is canceled by compensating $\mu$ dependence of $m^{2}, \lambda$, and field normalization. This is how the beta functions of $m^{2}, \lambda$ are derived. In the remainder of this paper we wish to do the same for the MS solutions of Polchinski's equation.

This is an appropriate place to comment on the work of Hughes and Liu [3]. They parameterize the action using three parameters:

$$
\begin{aligned}
\rho_{1} & \equiv \mathcal{V}_{2}(\Lambda ; 0,0) \\
\rho_{2} & \left.\equiv \frac{\partial}{\partial p^{2}} \mathcal{V}_{2}(\Lambda ; p,-p)\right|_{p=0} \\
\rho_{3} & \equiv \mathcal{V}_{4}(\Lambda ; 0,0,0,0)
\end{aligned}
$$

A solution to (11) is specified by the conditions at $\Lambda=\Lambda_{R}$ :

$$
\rho_{1}=\rho_{2}=0, \quad \rho_{3}=-\lambda
$$

$\Lambda_{R}$ obviously corresponds to our $\mu$. There are two problems. One, which is crucial, is that the dependence of the action on the choice of $\Lambda_{R}$ is neglected. The other is that this is not a mass independent scheme; the three parameters depend on $m^{2}$ non-trivially. Approximate mass independence is obtained by taking $\Lambda^{2}$ very large compared to $\mathrm{m}^{2}$. From the $\Lambda$ dependence of $\rho_{1,2,3}$, they have derived the beta function and anomalous dimensions at the lowest non-trivial orders. We will make a further remark in sect. 8 .

\section{Composite operators}

To derive the $\mu$ dependence of the action, we will use the technique of composite operators. (See, for example, [6].) This section contains a brief summary.

Let $\Phi(p)$ be a composite operator of momentum $p$ :

$$
\begin{aligned}
\Phi(p)=\sum_{n=1}^{\infty} & \frac{1}{(2 n) !} \int_{p_{1}, \cdots, p_{2 n}} \phi\left(p_{1}\right) \cdots \phi\left(p_{2 n}\right) \\
& \times(2 \pi)^{4} \delta^{(4)}\left(p_{1}+\cdots+p_{2 n}-p\right) \cdot \Phi_{2 n}\left(\Lambda ; p_{1}, \cdots, p_{2 n}\right)
\end{aligned}
$$


The $\Lambda$ dependence

$$
\begin{aligned}
-\Lambda \frac{\partial}{\partial \Lambda} \Phi(p)= & \int_{q} \frac{\Delta(q / \Lambda)}{q^{2}+m^{2}}\left(\frac{q^{2}+m^{2}}{K(q / \Lambda)} \phi(q) \frac{\delta}{\delta \phi(q)}\right. \\
& \left.+\frac{\delta S}{\delta \phi(-q)} \frac{\delta}{\delta \phi(q)}+\frac{1}{2} \frac{\delta^{2}}{\delta \phi(q) \delta \phi(-q)}\right) \Phi(p)
\end{aligned}
$$

guarantees that the correlation functions

$$
\left\langle\Phi(p) \phi\left(p_{1}\right) \cdots \phi\left(p_{2 n}\right)\right\rangle_{m^{2}, \lambda ; \mu} \equiv\left\langle\Phi(p) \phi\left(p_{1}\right) \cdots \phi\left(p_{2 n}\right)\right\rangle_{S(\Lambda)} \prod_{i=1}^{2 n} \frac{1}{K\left(p_{i} / \Lambda\right)}
$$

are independent of $\Lambda$. Composite operators of dimension $d$ satisfy the asymptotic conditions

$$
\Phi_{2 n>d}\left(\Lambda ; p_{1}, \cdots, p_{2 n}\right) \longrightarrow 0 \quad \text { as } \quad \Lambda \rightarrow \infty
$$

\subsection{Composite operators in the MS scheme}

For concreteness and also for later convenience, we consider scalar composite operators of dimension 2 and 4 . We will show how to construct composite operators using an analogous MS condition.

A dimension 2 operator $\Phi$ has the asymptotic behavior:

$$
\Phi_{2}\left(\Lambda ; p_{1}, p_{2}\right)=a_{2}(\ln (\Lambda / \mu))+\cdots
$$

where we suppress the $\lambda$ dependence of $a_{2}$, and the dots denote the part vanishing in the limit $\Lambda \rightarrow \infty$. In the MS scheme we define $\Phi$ by imposing the asymptotic condition:

$$
a_{2}(0)=1
$$

We will denote this operator as

$$
\left[\frac{1}{2} \phi^{2}\right]_{\mathrm{MS}}(p)
$$

Next we consider an operator $\Phi$ of dimension 4 . It must satisfy the following asymptotic behavior:

$$
\begin{aligned}
& \Phi_{2}\left(\Lambda ; p_{1}, p_{2}\right)= \Lambda^{2} a_{2}(\ln (\Lambda / \mu))+m^{2} b_{2}(\ln (\Lambda / \mu)) \\
&-\left(p_{1} p_{2}\right) c_{2}(\ln (\Lambda / \mu))-\left(p_{1}^{2}+p_{2}^{2}\right) c_{2}^{\prime}(\ln (\Lambda / \mu))+\cdots \\
& \Phi_{4}\left(\Lambda ; p_{1}, \cdots, p_{4}\right)=a_{4}(\ln (\Lambda / \mu))+\cdots
\end{aligned}
$$

In the MS scheme we can define three linearly independent operators as follows:

- $\left[\frac{1}{4 !} \phi^{4}\right]_{\mathrm{MS}}(p)$ satisfying

$$
a_{4}(0)=1, \quad c_{2}(0)=c_{2}^{\prime}(0)=b_{2}(0)=0
$$

- $\left[\frac{1}{2}\left(\partial_{\mu} \phi\right)^{2}\right]_{\mathrm{MS}}(p)$ satisfying

$$
c_{2}(0)=1, \quad a_{4}(0)=c_{2}^{\prime}(0)=b_{2}(0)=0
$$

- $\left[\phi \partial^{2} \phi\right]_{\mathrm{MS}}(p)$ satisfying

$$
c_{2}^{\prime}(0)=1, \quad a_{4}(0)=c_{2}(0)=b_{2}(0)=0
$$


For zero momentum we have only two linearly independent operators since

$$
\left[\frac{1}{2}\left(\partial_{\mu} \phi\right)^{2}\right]_{\mathrm{MS}}(0)=-\frac{1}{2}\left[\phi \partial^{2} \phi\right]_{\mathrm{MS}}(0)
$$

Hence, an arbitrary dimension 4 composite operator with zero momentum is given as

$$
\Phi=x m^{2}\left[\frac{1}{2} \phi^{2}\right]_{\mathrm{MS}}+y\left[\frac{1}{4 !} \phi^{4}\right]_{\mathrm{MS}}+z\left[\frac{1}{2}\left(\partial_{\mu} \phi\right)^{2}\right]_{\mathrm{MS}}
$$

where the $\Lambda$-independent coefficients can be extracted from the asymptotic behavior:

$$
x=b_{2}(0), \quad y=a_{4}(0), \quad z=\left(c_{2}-2 c_{2}^{\prime}\right)(0)
$$

\section{Composite operators in terms of the action $S$}

The scalar composite operators of dimension 2,4 with zero momentum are special in that they can be constructed directly out of the action $S$. It is easy to see why. $\left[\frac{1}{2} \phi^{2}\right]_{\mathrm{MS}}$ is the mass term, and it can be obtained essentially by differentiating $S$ with respect to $m^{2} \cdot\left[\frac{1}{4 !} \phi^{4}\right]_{\mathrm{MS}}$ is the interaction term, and is obtained by differentiating $S$ with respect to $\lambda$. The hard part is to construct $\left[\frac{1}{2}\left(\partial_{\mu} \phi\right)^{2}\right]_{\mathrm{MS}}$; we need to use the equation of motion.

We examine three cases one by one. For later convenience we introduce the following expansions in $m^{2}$ :

$$
\begin{aligned}
& \mathcal{V}_{4}(\Lambda ; p,-p, 0,0)=A_{4}(\ln (\Lambda / \mu) ; p / \Lambda)+\frac{m^{2}}{\Lambda^{2}} B_{4}(\ln (\Lambda / \mu) ; p / \Lambda)+\cdots \\
& \mathcal{V}_{6}(\Lambda ; p,-p, 0, \cdots, 0)=\frac{1}{\Lambda^{2}} A_{6}(\ln (\Lambda / \mu) ; p / \Lambda)+\cdots
\end{aligned}
$$

where $p$ is considered of order $\Lambda$.

\section{1. $\mathcal{O}_{m}$}

We define

$$
\begin{aligned}
\mathcal{O}_{m} & \equiv-\frac{\partial S}{\partial m^{2}} \\
& -\int_{q} \frac{K(q / \Lambda)(1-K(q / \Lambda))}{\left(q^{2}+m^{2}\right)^{2}} \frac{1}{2}\left(\frac{\delta S}{\delta \phi(q)} \frac{\delta S}{\delta \phi(-q)}+\frac{\delta^{2} S}{\delta \phi(q) \delta \phi(-q)}\right)
\end{aligned}
$$

From the asymptotic behavior, we obtain

$$
\mathcal{O}_{m}=x_{m}\left[\frac{1}{2} \phi^{2}\right]_{\mathrm{MS}}
$$

where

$$
x_{m} \equiv 1-\frac{1}{2} \int_{q} \frac{K(q)(1-K(q))}{q^{4}} A_{4}(0 ; q)
$$

In the appendix we derive

$$
\left\langle\mathcal{O}_{m} \phi\left(p_{1}\right) \cdots \phi\left(p_{2 n}\right)\right\rangle_{m^{2}, \lambda ; \mu}=-\frac{\partial}{\partial m^{2}}\left\langle\phi\left(p_{1}\right) \cdots \phi\left(p_{2 n}\right)\right\rangle_{m^{2}, \lambda ; \mu}
$$


6.2. $\mathcal{O}_{\lambda}$

We define

$$
\mathcal{O}_{\lambda} \equiv-\frac{\partial S}{\partial \lambda}
$$

From the definition, it is straightforward to show

$$
\left\langle\mathcal{O}_{\lambda} \phi\left(p_{1}\right) \cdots \phi\left(p_{2 n}\right)\right\rangle_{m^{2}, \lambda ; \mu}=-\frac{\partial}{\partial \lambda}\left\langle\phi\left(p_{1}\right) \cdots \phi\left(p_{2 n}\right)\right\rangle_{m^{2}, \lambda ; \mu}
$$

From the asymptotic behavior, we obtain

$$
\mathcal{O}_{\lambda}=\left[\frac{1}{4 !} \phi^{4}\right]_{\mathrm{MS}}
$$

\section{3. $\mathcal{N}$}

We define

$$
\begin{aligned}
\mathcal{N} & \equiv-\int_{q} \phi(q) \frac{\delta S}{\delta \phi(q)} \\
& -\int_{q} \frac{K(q / \Lambda)(1-K(q / \Lambda))}{q^{2}+m^{2}}\left(\frac{\delta S}{\delta \phi(q)} \frac{\delta S}{\delta \phi(-q)}+\frac{\delta^{2} S}{\delta \phi(q) \delta \phi(-q)}\right)
\end{aligned}
$$

We show, in the appendix, that

$$
\left\langle\mathcal{N} \phi\left(p_{1}\right) \cdots \phi\left(p_{2 n}\right)\right\rangle_{m^{2}, \lambda ; \mu}=2 n\left\langle\phi\left(p_{1}\right) \cdots \phi\left(p_{2 n}\right)\right\rangle_{m^{2}, \lambda ; \mu}
$$

By examining the asymptotic behavior we obtain

$$
\mathcal{N}=x_{\mathcal{N}} m^{2}\left[\frac{1}{2} \phi^{2}\right]_{\mathrm{MS}}+y_{\mathcal{N}}\left[\frac{1}{4 !} \phi^{4}\right]_{\mathrm{MS}}+z_{\mathcal{N}}\left[\frac{1}{2}\left(\partial_{\mu} \phi\right)^{2}\right]_{\mathrm{MS}}
$$

where

$$
\begin{aligned}
& x_{\mathcal{N}} \equiv 2+\int_{q} K(q)(1-K(q))\left(-\frac{B_{4}(0 ; q)}{q^{2}}+\frac{A_{4}(0 ; q)}{q^{4}}\right) \\
& y_{\mathcal{N}} \equiv-4 \lambda-\int_{q} \frac{K(q)(1-K(q))}{q^{2}} A_{6}(0 ; q) \\
& z_{\mathcal{N}} \equiv 2-\int_{q} \frac{K(q)(1-K(q))}{q^{2}} C_{4}(0 ; q)
\end{aligned}
$$

In (70) $C_{4}$ is defined by

$$
\left.\frac{1}{\Lambda^{2}} \delta_{\mu \nu} C_{4}(\ln (\Lambda / \mu) ; p / \Lambda) \equiv \frac{1}{2} \frac{\partial^{2}}{\partial q_{\mu} \partial q_{\nu}} \mathcal{V}_{4}(\Lambda ; p,-p, q,-q)\right|_{m^{2}=q^{2}=0}
$$

where the angular average over $p_{\mu}$ is taken on the right-hand side. Using the results for $\mathcal{O}_{m}$ and $\mathcal{O}_{\lambda}$, we can rewrite this as

$$
\mathcal{N}=\frac{x_{\mathcal{N}}}{x_{m}} m^{2} \mathcal{O}_{m}+y_{\mathcal{N}} \mathcal{O}_{\lambda}+z_{\mathcal{N}}\left[\frac{1}{2}\left(\partial_{\mu} \phi\right)^{2}\right]_{\mathrm{MS}}
$$


To conclude this section, we have shown that the three scalar operators of dimension 2, 4 can be constructed from $S$ as

$$
\begin{aligned}
{\left[\frac{1}{2} \phi^{2}\right]_{\mathrm{MS}} } & =\frac{1}{x_{m}} \mathcal{O}_{m} \\
{\left[\frac{1}{4 !} \phi^{4}\right]_{\mathrm{MS}} } & =\mathcal{O}_{\lambda} \\
{\left[\frac{1}{2}\left(\partial_{\mu} \phi\right)^{2}\right]_{\mathrm{MS}} } & =\frac{1}{z_{\mathcal{N}}}\left(\mathcal{N}-\frac{x_{\mathcal{N}}}{x_{m}} m^{2} \mathcal{O}_{m}-y_{\mathcal{N}} \mathcal{O}_{\lambda}\right)
\end{aligned}
$$

\section{Beta function and anomalous dimensions}

With all the necessary tools in our hands, we are ready to derive the ordinary RG equations for the renormalized correlation functions. This is done by considering the $\mu$ dependence of the action:

$$
\Psi \equiv \mu \frac{\partial}{\partial \mu} S\left(\Lambda ; m^{2}, \lambda ; \mu\right)
$$

Differentiating Polchinski's equation with respect to $\mu$, we obtain

$$
\begin{aligned}
-\Lambda \frac{\partial}{\partial \Lambda} \Psi= & \int_{q} \frac{\Delta(q / \Lambda)}{q^{2}+m^{2}}\left[\frac{q^{2}+m^{2}}{K(q / \Lambda)} \phi(q) \frac{\delta}{\delta \phi(q)}\right. \\
& \left.+\frac{\delta S}{\delta \phi(-q)} \frac{\delta}{\delta \phi(q)}+\frac{1}{2} \frac{\delta^{2}}{\delta \phi(q) \delta \phi(-q)}\right] \Psi
\end{aligned}
$$

Hence, $\Psi$ is a composite operator of zero momentum. This has dimension 4 , and hence must be a linear combination of three independent operators $\mathcal{O}_{m}, \mathcal{O}_{\lambda}, \mathcal{N}$.

We first expand $\Psi$ in terms of MS operators by examining its asymptotic behaviors. We find

$$
\begin{aligned}
& \Psi_{2}(\Lambda ; p,-p)=\Lambda^{2} \dot{a}_{2}(\ln (\Lambda / \mu) ; \lambda) \\
& +m^{2} \dot{b}_{2}(\ln (\Lambda / \mu) ; \lambda)+p^{2} \dot{c}_{2}(\ln (\Lambda / \mu) ; \lambda)+\cdots \\
& \Psi_{4}\left(\Lambda ; p_{1}, \cdots, p_{4}\right)=\dot{a}_{4}(\ln (\Lambda / \mu) ; \lambda)+\cdots
\end{aligned}
$$

where

$$
\dot{b}_{2}(\ln (\Lambda / \mu) ; \lambda) \equiv \mu \frac{\partial}{\partial \mu} b_{2}(\ln (\Lambda / \mu) ; \lambda)=-\Lambda \frac{\partial}{\partial \Lambda} b_{2}(\ln (\Lambda / \mu) ; \lambda), \cdots
$$

are the derivatives of the coefficients introduced in sect. 4. Hence, $\Psi$ can be expanded as

where

$$
\Psi=\dot{b}_{2}(\lambda) m^{2}\left[\frac{1}{2} \phi^{2}\right]_{\mathrm{MS}}+\dot{c}_{2}(\lambda)\left[\frac{1}{2}\left(\partial_{\mu} \phi\right)^{2}\right]_{\mathrm{MS}}+\dot{a}_{4}(\lambda)\left[\frac{1}{4 !} \phi^{4}\right]_{\mathrm{MS}}
$$

$$
\dot{b}_{2}(\lambda) \equiv \dot{b}_{2}(0 ; \lambda), \quad \dot{c}_{2}(\lambda) \equiv \dot{c}_{2}(0 ; \lambda), \quad \dot{a}_{4}(\lambda) \equiv \dot{a}_{4}(0 ; \lambda)
$$

Using the results of the previous section, we can rewrite the above using $\mathcal{O}_{m}, \mathcal{O}_{\lambda}$, and $\mathcal{N}$ instead:

$$
\begin{aligned}
\Psi & =\frac{\dot{b}_{2}}{x_{m}} m^{2} \mathcal{O}_{m}+\frac{\dot{c}_{2}}{z_{\mathcal{N}}}\left(\mathcal{N}-\frac{x_{\mathcal{N}}}{x_{m}} m^{2} \mathcal{O}_{m}-y_{\mathcal{N}} \mathcal{O}_{\lambda}\right)+\dot{a}_{4} \mathcal{O}_{\lambda} \\
& =\frac{1}{x_{m}}\left(\dot{b}_{2}-\dot{c}_{2} \frac{x_{\mathcal{N}}}{z_{\mathcal{N}}}\right) m^{2} \mathcal{O}_{m}+\left(\dot{a}_{4}-\dot{c}_{2} \frac{y_{\mathcal{N}}}{z_{\mathcal{N}}}\right) \mathcal{O}_{\lambda}+\frac{1}{z_{\mathcal{N}}} \dot{c}_{2} \mathcal{N}
\end{aligned}
$$


We now define

$$
\begin{aligned}
\beta(\lambda) & \equiv-\left(\dot{a}_{4}-\dot{c}_{2} \frac{y_{\mathcal{N}}}{z_{\mathcal{N}}}\right) \\
\beta_{m}(\lambda) & \equiv-\frac{1}{x_{m}}\left(\dot{b}_{2}-\dot{c}_{2} \frac{x_{\mathcal{N}}}{z_{\mathcal{N}}}\right) \\
\gamma(\lambda) & \equiv-\frac{1}{z_{N}} \dot{c}_{2}
\end{aligned}
$$

so that

$$
\Psi \equiv \mu \frac{\partial S}{\partial \mu}=-\left(\beta_{m} m^{2} \mathcal{O}_{m}+\beta \mathcal{O}_{\lambda}+\gamma \mathcal{N}\right)
$$

It is now trivial to derive the $\mu$ dependence of the correlation functions:

$$
\begin{aligned}
& \mu \frac{\partial}{\partial \mu}\left\langle\phi\left(p_{1}\right) \cdots \phi\left(p_{2 n}\right)\right\rangle_{m^{2}, \lambda ; \mu} \\
& =\left\langle\mu \frac{\partial S}{\partial \mu} \phi\left(p_{1}\right) \cdots \phi\left(p_{2 n}\right)\right\rangle_{m^{2}, \lambda ; \mu} \\
& =\left\langle\left(-m^{2} \beta_{m} \mathcal{O}_{m}-\beta \mathcal{O}_{\lambda}-\gamma \mathcal{N}\right) \phi\left(p_{1}\right) \cdots \phi\left(p_{2 n}\right)\right\rangle_{m^{2}, \lambda ; \mu} \\
& =\left(m^{2} \beta_{m} \frac{\partial}{\partial m^{2}}+\beta \frac{\partial}{\partial \lambda}-2 n \gamma\right)\left\langle\phi\left(p_{1}\right) \cdots \phi\left(p_{2 n}\right)\right\rangle_{m^{2}, \lambda ; \mu}
\end{aligned}
$$

where we have used (61,63,66). This is nothing but the usual $R G$ equations for the renormalized correlation functions. Hence, $\beta$ is the beta function of $\lambda$, and $\beta_{m}, \gamma$ are the anomalous dimensions of $m^{2}, \phi$, respectively.

At 1-loop, we find

$$
\dot{b}_{2}(\lambda)=\frac{\lambda}{(4 \pi)^{2}}, \quad \dot{c}_{2}(\lambda)=0, \quad \dot{a}_{4}(\lambda)=3 \frac{\lambda^{2}}{(4 \pi)^{2}}
$$

We also find, to leading order in $\lambda$,

$$
x_{m}=1, \quad x_{\mathcal{N}}=2, \quad y_{\mathcal{N}}=-4 \lambda, \quad z_{\mathcal{N}}=2
$$

Hence,

$$
\beta=-3 \frac{\lambda^{2}}{(4 \pi)^{2}}, \quad \beta_{m}=-\frac{\lambda}{(4 \pi)^{2}}, \quad \gamma=0
$$

reproducing the familiar results. For two-loop calculations, see [4] (and [3] for $\gamma$ ).

\section{Simplification in the Wegner-Houghton limit}

In the Wegner-Houghton limit [8] we take

$$
K(x)=\theta\left(1-x^{2}\right)= \begin{cases}1 & \left(x^{2}<1\right) \\ 0 & \left(x^{2}>1\right)\end{cases}
$$

This limit is known to introduce non-locality to the theory. For example, the inverse Fourier transform of the high-momentum propagator

$$
\int_{p} \mathrm{e}^{i p r} \frac{1-\theta\left(\Lambda^{2}-p^{2}\right)}{p^{2}+m^{2}}
$$


behaves as $\sin (\Lambda r) / r^{2}$ for $\Lambda r \gg 1$. Despite this, this limit has its own merit of bringing nice simplification to ERG.

In the Wegner-Houghton limit we obtain

$$
K(p / \Lambda)(1-K(p / \Lambda))=0
$$

Hence, $\mathcal{O}_{m}, \mathcal{O}_{\lambda}, \mathcal{N}$ become simply

$$
\mathcal{O}_{m}=-\frac{\partial S}{\partial m^{2}}, \quad \mathcal{O}_{\lambda}=-\frac{\partial S}{\partial \lambda}, \quad \mathcal{N}=-\int_{p} \phi(p) \frac{\delta S}{\delta \phi(p)}
$$

Therefore, (88) gets simplified to

$$
\mu \frac{\partial S}{\partial \mu}=\left(\beta_{m} m^{2} \frac{\partial}{\partial m^{2}}+\beta \frac{\partial}{\partial \lambda}+\gamma \int_{p} \phi(p) \frac{\delta}{\delta \phi(p)}\right) S
$$

meaning that a change of $\mu$ can be compensated by appropriate changes in $m^{2}, \lambda$ and field normalization. By examining the asymptotic behavior of this equation, we obtain the following equations:

$$
\begin{aligned}
& \left(\mu \frac{\partial}{\partial \mu}-\beta \partial_{\lambda}\right)\left(1-b_{2}(\ln (\Lambda / \mu) ; \lambda)\right)=\left(\beta_{m}+2 \gamma\right) \cdot\left(1-b_{2}(\ln (\Lambda / \mu) ; \lambda)\right) \\
& \left(\mu \frac{\partial}{\partial \mu}-\beta \partial_{\lambda}\right)\left(1-c_{2}(\ln (\Lambda / \mu) ; \lambda)\right)=2 \gamma \cdot\left(1-c_{2}(\ln (\Lambda / \mu) ; \lambda)\right) \\
& \left(\mu \frac{\partial}{\partial \mu}-\beta \partial_{\lambda}\right) a_{4}(\ln (\Lambda / \mu) ; \lambda)=4 \gamma \cdot a_{4}(\ln (\Lambda / \mu) ; \lambda)
\end{aligned}
$$

We introduce a running coupling $\bar{\lambda}(t ; \lambda)$ by

$$
\frac{\partial}{\partial t} \bar{\lambda}(t ; \lambda)=\beta(\bar{\lambda}(t ; \lambda)), \quad \bar{\lambda}(0 ; \lambda)=\lambda
$$

so that the above equations are solved by

$$
\begin{aligned}
& \left.\left.1-b_{2}(\ln (\Lambda / \mu) ; \lambda)=\exp \left[-\int_{0}^{\ln (\Lambda / \mu)} d t\left\{\beta_{m}(\bar{\lambda}(-t ; \lambda))+2 \gamma(\bar{\lambda}(-t ; \lambda)\}\right\}\right\} \mid\right\} 2\right) \\
& 1-c_{2}(\ln (\Lambda / \mu) ; \lambda)=\exp \left[-2 \int_{0}^{\ln (\Lambda / \mu)} d t \gamma(\bar{\lambda}(-t ; \lambda))\right] \\
& a_{4}(\ln (\Lambda / \mu) ; \lambda)=-\exp \left[-4 \int_{0}^{\ln (\Lambda / \mu)} d t \gamma(\bar{\lambda}(-t ; \lambda))\right] \bar{\lambda}(-\ln (\Lambda / \mu)(\mathbb{A} 04)
\end{aligned}
$$

Hence, the asymptotic behavior of the vertices are expressed fully in terms of $\beta, \beta_{m}, \gamma$. Conversely, we can determine $\beta, \beta_{m}, \gamma$ by calculating $b_{2}, c_{2}, a_{4}$. At the lowest non-trivial orders in $\lambda$, the above equations are valid for any choice of $K$. This explains why the correct results were obtained in [3]. (Their $\rho_{1,2,3}$ correspond to $b_{2}, c_{2}, a_{4}$, respectively.)

The above results are so similar to the well known relation for the renormalization constants in the MS scheme for dimensional regularization. [7] From this, we conjecture that $\beta, \beta_{m}, \gamma$ in the Wegner-Houghton limit are the same to all orders in $\lambda$ to those in the MS scheme for dimensional regularization. This is supported by the 2-loop calculations of $\gamma, \beta_{m}$ which are scheme dependent. [3, 4] But we have no other justification for this conjecture. 


\section{Conclusions}

In this paper we have explained how the ordinary RG equations arise from Polchinski's ERG equations. We achieved this goal in two steps:

(i) introduction of MS scheme - We characterize the solutions to ERG differential equations by their asymptotic behavior at large cutoff. A renormalization scale $\mu$ is introduced to organize the logarithmic dependence of the asymptotic vertices on the cutoff.

(ii) derivation of the $\mu$ dependence of the solution to ERG - We have shown that the $\mu$ derivative of the action is a composite operator of zero momentum, and therefore it can be expanded by the three operators whose properties we understand very well.

Throughout the paper we have emphasized the difference between Wilson's ERG and Polchinski's ERG. They share the same spirit, but there are crucial differences that make the derivation of $\beta, \beta_{m}, \gamma$ somewhat non-trivial for Polchinski's ERG.

Is there any way of modifying Polchinski's ERG to get something more like Wilson's? There is. Instead of following the $\Lambda$ dependence of $S\left(\Lambda ; m^{2}, \lambda ; \mu\right)$, we follow the $\mu$ dependence of

$$
\bar{S}\left(\mu ; m^{2}, \lambda\right) \equiv S\left(\mu ; m^{2}, \lambda ; \mu\right)
$$

This $\bar{S}$ is characterized by the large $\mu$ behaviors

$$
\begin{aligned}
& \overline{\mathcal{V}}_{2}\left(\mu ; m^{2}, \lambda ; p,-p\right)=\mu^{2} a_{2}(\lambda)+\cdots \\
& \overline{\mathcal{V}}_{4}\left(\mu ; m^{2}, \lambda ; p_{1}, \cdots, p_{4}\right)=-\lambda+\cdots \\
& \overline{\mathcal{V}}_{2 n \geq 6}\left(\mu ; m^{2}, \lambda ; p_{1}, \cdots, p_{2 n}\right)=\cdots
\end{aligned}
$$

which are devoid of logarithms. (Although the asymptotic parts have no explicit $\mu$ dependence, the parts vanishing asymptotically depend on $\mu$. This can be seen from the two one-loop examples (30, 32).) Under an infinitesimal change of $\mu \rightarrow \mu(1-\Delta t)$, we also change $m^{2}, \lambda$ as

$$
m^{2} \longrightarrow m^{2}\left(1+\Delta t \cdot \beta_{m}\right), \quad \lambda \longrightarrow \lambda+\Delta t \cdot \beta
$$

Then, the new ERG differential equation gives

$$
\begin{aligned}
\left(-\mu \frac{\partial}{\partial \mu}+\beta_{m}(\lambda) m^{2}\right. & \frac{\partial}{\partial m^{2}}+\beta(\lambda) \frac{\partial}{\partial \lambda} \\
& \left.+\gamma(\lambda) \int_{p} \phi(p) \frac{\delta}{\delta \phi(p)}\right) \bar{S}\left(\mu ; m^{2}, \lambda\right)
\end{aligned}
$$

in terms of integrals over momenta of order $\mu$. (This has been done in [4].) By construction,

$$
\bar{S}\left(\mu(1-\Delta t) ; m^{2}\left(1+\Delta t \beta_{m}\right), \lambda+\Delta t \beta\right)
$$

lies on the same ERG trajectory as $\bar{S}\left(\mu ; m^{2}, \lambda\right)$. 
As we have seen in this paper, the technique of composite operators is a useful and perhaps essential tool for addressing formal questions about perturbative ERG. The composite operators have already been shown to play essential roles in the applications of ERG to gauge theories and non-linear sigma models. (See for example, [6].) This is also the case in a forthcoming paper [9] where we will apply the MS scheme of ERG to QED.

\section{Appendix A. Derivation of $\mathcal{O}_{m}$ and $\mathcal{N}$}

In this appendix we wish to derive the relation of the operators $\mathcal{O}_{m}, \mathcal{O}_{\lambda}, \mathcal{N}$ to the action. As preparation, we introduce two types of operators. (We call them operators though they are not necessarily composite operators on their own.)

Appendix A.1. Type 1 operator $\mathcal{O}_{1}[f]$

We define

$$
\mathcal{O}_{1}[f] \equiv \int_{p} f(p) \phi(p) \frac{\delta S}{\delta \phi(p)}
$$

where $f(p)$ is an arbitrary function of momentum. This generates an infinitesimal linear change of field:

$$
\delta \phi(p)=-f(p) \phi(p)
$$

It is straightforward to derive the correlation functions of $\mathcal{O}_{1}$ :

$$
\begin{aligned}
& \left\langle\mathcal{O}_{1}[f] \phi\left(p_{1}\right) \cdots \phi\left(p_{2 n}\right)\right\rangle_{S} \cdot(2 \pi)^{4} \delta^{(4)}\left(p_{1}+\cdots+p_{2 n}\right) \\
& =\int_{p} f(p) \int[d \phi] \phi(p) \frac{\delta S}{\delta \phi(p)} \phi\left(p_{1}\right) \cdots \phi\left(p_{2 n}\right) \mathrm{e}^{S} \\
& =\int_{p} f(p) \int[d \phi] \phi(p) \phi\left(p_{1}\right) \cdots \phi\left(p_{2 n}\right) \frac{\delta \mathrm{e}^{S}}{\delta \phi(p)} \\
& =-\int_{p} f(p) \int[d \phi] \phi(p) \frac{\delta}{\delta \phi(p)}\left(\phi\left(p_{1}\right) \cdots \phi\left(p_{2 n}\right)\right) \mathrm{e}^{S} \\
& =-\sum_{i=1}^{2 n} f\left(p_{i}\right) \cdot\left\langle\phi\left(p_{1}\right) \cdots \phi\left(p_{2 n}\right)\right\rangle_{S} \cdot(2 \pi)^{4} \delta^{(4)}\left(p_{1}+\cdots+p_{2 n}\right)
\end{aligned}
$$

where we have neglected the derivative acting on $\phi(p)$, since it does not contribute to the connected part of the correlation function. Thus, we obtain

$$
\left\langle\mathcal{O}_{1}[f] \phi\left(p_{1}\right) \cdots \phi\left(p_{2 n}\right)\right\rangle_{S}=-\sum_{i=1}^{2 n} f\left(p_{i}\right) \cdot\left\langle\phi\left(p_{1}\right) \cdots \phi\left(p_{2 n}\right)\right\rangle_{S}
$$

Appendix A.2. $\mathcal{O}_{2}[C]$

We define

$$
\mathcal{O}_{2}[C] \equiv \int_{p} C(p) \frac{1}{2}\left(\frac{\delta S}{\delta \phi(p)} \frac{\delta S}{\delta \phi(-p)}+\frac{\delta^{2} S}{\delta \phi(p) \delta \phi(-p)}\right)
$$


where $C(p)=C(-p)$. This generates an infinitesimal non-linear change of field:

$$
\delta \phi(p)=-C(p) \frac{\delta S}{\delta \phi(-p)}
$$

We can show

$$
\begin{aligned}
\left\langle\mathcal{O}_{2}[C] \phi(p) \phi(-p)\right\rangle_{S} & =C(p) \\
\left\langle\mathcal{O}_{2}[C] \phi\left(p_{1}\right) \cdots \phi\left(p_{2 n}\right)\right\rangle_{S} & =0 \quad(n>1)
\end{aligned}
$$

The first equation is derived as follows:

$$
\begin{aligned}
& \left\langle\mathcal{O}_{2}[C] \phi\left(p_{1}\right) \phi\left(p_{2}\right)\right\rangle_{S} \cdot(2 \pi)^{4} \delta^{(4)}\left(p_{1}+p_{2}\right) \\
& =\int_{q} C(q) \frac{1}{2} \int[d \phi]\left(\frac{\delta S}{\delta \phi(q)} \frac{\delta S}{\delta \phi(-q)}+\frac{\delta^{2} S}{\delta \phi(q) \delta \phi(-q)}\right) \phi\left(p_{1}\right) \phi\left(p_{2}\right) \mathrm{e}^{S} \\
& =\int_{q} C(q) \frac{1}{2} \int[d \phi] \phi\left(p_{1}\right) \phi\left(p_{2}\right) \frac{\delta^{2} \mathrm{e}^{S}}{\delta \phi(q) \delta \phi(-q)} \\
& =\int_{q} C(q) \frac{1}{2} \int[d \phi] \mathrm{e}^{S} \frac{\delta^{2}}{\delta \phi(q) \delta \phi(-q)} \phi\left(p_{1}\right) \phi\left(p_{2}\right) \\
& =C\left(p_{1}\right)(2 \pi)^{4} \delta^{(4)}\left(p_{1}+p_{2}\right)
\end{aligned}
$$

For $n>1$, the connected part receives no contribution.

We note that the right-hand side of Polchinski's ERG equation (11) is the sum of type $1 \& 2$ operators.

Appendix A.3. Derivation of $\mathcal{O}_{m}$

We first consider the two-point function. Since

$$
\langle\phi(p) \phi(-p)\rangle_{m^{2}, \lambda} \equiv \frac{1-1 / K(p / \Lambda)}{p^{2}+m^{2}}+\langle\phi(p) \phi(-p)\rangle_{S} \cdot \frac{1}{K(p / \Lambda)^{2}}
$$

we obtain

$$
\begin{aligned}
& -\frac{\partial}{\partial m^{2}}\langle\phi(p) \phi(-p)\rangle_{m^{2}, \lambda} \\
& =\frac{1-1 / K(p / \Lambda)}{\left(p^{2}+m^{2}\right)^{2}}-\left\langle\phi(p) \phi(-p) \frac{\partial S}{\partial m^{2}}\right\rangle_{S} \frac{1}{K(p / \Lambda)^{2}} \\
& =\left(-\frac{K(p / \Lambda)(1-K(p / \Lambda))}{\left(p^{2}+m^{2}\right)^{2}}-\left\langle\phi(p) \phi(-p) \frac{\partial S}{\partial m^{2}}\right\rangle_{S}\right) \frac{1}{K(p / \Lambda)^{2}}
\end{aligned}
$$

For the higher-point functions, we simply obtain

$$
\begin{aligned}
- & \frac{\partial}{\partial m^{2}}\left\langle\phi\left(p_{1}\right) \cdots \phi\left(p_{2 n}\right)\right\rangle_{m^{2}, \lambda} \\
& =-\left\langle\phi\left(p_{1}\right) \cdots \phi\left(p_{2 n}\right) \frac{\partial S}{\partial m^{2}}\right\rangle_{S} \cdot \prod_{i=1}^{2 n} \frac{1}{K\left(p_{i} / \Lambda\right)}
\end{aligned}
$$

Thus, using a type 2 operator $\mathcal{O}_{2}[C]$ for

$$
C(p) \equiv \frac{K(p / \Lambda)(1-K(p / \Lambda))}{\left(p^{2}+m^{2}\right)}
$$


we obtain

$$
\begin{aligned}
- & \frac{\partial}{\partial m^{2}}\left\langle\phi\left(p_{1}\right) \cdots \phi\left(p_{2 n}\right)\right\rangle_{m^{2}, \lambda} \\
& =\left\langle\phi\left(p_{1}\right) \cdots \phi\left(p_{2 n}\right)\left(-\frac{\partial S}{\partial m^{2}}-\mathcal{O}_{2}[C]\right)\right\rangle_{m^{2}, \lambda}
\end{aligned}
$$

for any $n=1,2, \cdots$. This defines $\mathcal{O}_{m}$.

\section{Appendix A.4. Derivation of $\mathcal{N}$}

We consider the type 1 operator $\mathcal{O}_{1}[f]$ corresponding to $f=-1$ :

$$
\mathcal{O}_{1}[f]=-\int_{p} \phi(p) \frac{\delta S}{\delta \phi(p)}
$$

Then, we obtain

$$
\left\langle\mathcal{O}_{1}[f] \phi\left(p_{1}\right) \cdots \phi\left(p_{2 n}\right)\right\rangle_{S}=2 n\left\langle\phi\left(p_{1}\right) \cdots \phi\left(p_{2 n}\right)\right\rangle_{S}
$$

for any $n$. On the other hand, we have

$$
\begin{gathered}
2\langle\phi(p) \phi(-p)\rangle_{m^{2}, \lambda}=-\frac{2(1-1 / K(p / \Lambda))}{p^{2}+m^{2}}+2\langle\phi(p) \phi(-p)\rangle_{S} \cdot \frac{1}{K(p / \Lambda)^{2}} \\
=\left(-\frac{2 K(p / \Lambda)(1-K(p / \Lambda))}{p^{2}+m^{2}}+\left\langle\phi(p) \phi(-p) \mathcal{O}_{1}[f]\right\rangle_{S}\right) \frac{1}{K(p / \Lambda)^{2}}
\end{gathered}
$$

and

$$
\begin{aligned}
2 n & \left\langle\phi\left(p_{1}\right) \cdots \phi\left(p_{2 n}\right)\right\rangle_{m^{2}, \lambda}=2 n\left\langle\phi\left(p_{1}\right) \cdots \phi\left(p_{2 n}\right)\right\rangle_{S} \cdot \prod_{i=1}^{2 n} \frac{1}{K\left(p_{i} / \Lambda\right)} \\
& =\left\langle\phi\left(p_{1}\right) \cdots \phi\left(p_{2 n}\right) \mathcal{O}_{1}[f]\right\rangle_{S} \cdot \prod_{i=1}^{2 n} \frac{1}{K\left(p_{i} / \Lambda\right)}
\end{aligned}
$$

for $n>1$. Thus, using a type 2 operator $\mathcal{O}_{2}[C]$ for

$$
C(p) \equiv-\frac{2 K(p / \Lambda)(1-K(p / \Lambda))}{p^{2}+m^{2}}
$$

we obtain

$$
2 n\left\langle\phi\left(p_{1}\right) \cdots \phi\left(p_{2 n}\right)\right\rangle_{m^{2}, \lambda}=\left\langle\phi\left(p_{1}\right) \cdots \phi\left(p_{2 n}\right)\left(\mathcal{O}_{1}[f]+\mathcal{O}_{2}[C]\right)\right\rangle_{m^{2}, \lambda}
$$

for any $n=1,2, \cdots$. This defines $\mathcal{N}$.

\section{References}

[1] Wilson K G and Kogut J 1974 Phys. Rept. 1275

[2] Polchinski J 1984 Nucl. Phys. B231 269

[3] Hughes J and Liu J 1988 Nucl. Phys. B307 183

[4] Sonoda H 2003 Beta functions in the integral equation approach to ERG Preprint hep-th/0302044

[5] Sonoda H 2003 Phys. Rev. D67 065011

[6] Becchi C 1991 On the construction of renormalized theories using renormalization group techniques (Parma lectures) Preprint hep-th/9607188

[7] 't Hooft G 1973 Nucl. Phys. B61 455

[8] Wegner F and Houghton A 1973 Phys. Rev. A8 401

[9] Sonoda H 2007 On the construction of QED using ERG Paper in preparation 